Service social

\title{
Groupe et prévention de l'agression chez les jeunes en milieu scolaire
}

\section{Yvan Tremblay et Alice M. Home}

Volume 39, numéro 1, 1990

Le groupe ici et ailleurs

URI : https://id.erudit.org/iderudit/706460ar

DOI : https://doi.org/10.7202/706460ar

Aller au sommaire du numéro

Éditeur(s)

École de service social de l'Université Laval

ISSN

1708-1734 (numérique)

Découvrir la revue

Citer cet article

Tremblay, Y. \& Home, A. M. (1990). Groupe et prévention de l'agression chez les jeunes en milieu scolaire. Service social, 39(1), 114-137.

https://doi.org/10.7202/706460ar
Résumé de l'article

Les comportements agressifs chez les jeunes et dans la société en général ont des répercussions sérieuses et un lourd coût social. Le groupe est un outil de prévention et d'intervention auprès des jeunes.

Cet article présente une expérience-pilote réalisée auprès de jeunes agressifs et prosociaux réunis dans un même groupe, dans le cadre d'un projet visant à prévenir la manifestation de comportements jugés destructeurs. On analyse ici les changements observés chez les membres en regard des objectifs du projet. 
Yvan Tremblay, étudiant à la maîtrise, École de service social, Université de Montréal.

Alice M. Home, professeure à l'École de service social, Université de Montréal.

\section{Groupe et prévention de l'agression chez les jeunes en milieu scolaire}

Yvan Tremblay Alice M. Home

Les comportements agressifs chez les jeunes et dans la société en général s'exprimant par le racisme, les insultes, l'extorsion et les agressions sexuelles, ont des répercussions sérieuses et un coût social inestimable. Femmes battues, enfants négligés, maltraités et abusés sexuellement témoignent de l'urgence de prévenir plutôt que de guérir. Le groupe est un outil de prévention qui peut permettre d'intervenir adéquatement auprès des jeunes avant que le problème ne se manifeste ou au moins très tôt après son apparition (Spain, 1986; Home et Darveau-Fournier, 1980).

L'intervention de groupe discutée dans cet article a fait l'objet d'un stage de maîtrise en service social et s'insère dans une recherche-action de type projet pilote. Le projet vise à prévenir la manifestation de comportements agressifs jugés destructeurs chez les jeunes (Hébert, 1987). L'évaluation de l'intervention occupait une place importante au sein du projet. L'objectif de cet article est d'analyser les changements observés chez les membres en rapport avec les objectifs du projet.

Nous décrirons d'abord le contexte de l'intervention de groupe en le situant dans le projet de prévention avant d'aborder la problématique de l'agression chez les jeunes. Nous discuterons du groupe comme moyen et outil de prévention des comportements agressifs en l'illustrant avec quelques expériences de prévention en groupe auprès des jeunes. Nous expliquerons la méthodologie utilisée afin d'apprécier les changements observés chez les membres. 
Enfin, nous présenterons et analyserons les résultats en rapport avec les objectifs poursuivis par le projet.

\section{Contexte et description de l'intervention}

L'intervention de groupe en milieu scolaire s'est déroulée de janvier à juin 1989. Cette intervention s'intégrait dans le projet de Hébert (1987) qui avait pour but l'implantation et l'évaluation d'un programme préventif auprès de jeunes agressifs et prosociaux dans une communauté locale. Le projet a eu lieu sous le parrainage de "Jeunes en flèche ", un organisme de parents bénévoles dont la vocation est de mettre sur pied des activités pour les jeunes qui fréquentent les écoles du quartier.

On entend par agressif un jeune qui adopte des comportements jugés destructeurs (détruire des objets, insulter, menacer, frapper quelqu'un, etc.), tandis qu'un jeune prosocial est celui qui a davantage de comportements positifs (offrir de l'aide, partager, etc.). Les jeunes ont été identifiés comme tels d'une part par le milieu scolaire et d'autre part par le milieu familial. Le projet prévoyait que les jeunes identifiés comme prosociaux soient majoritaires dans le groupe, selon un ratio de trois " prosociaux " pour un " agressif ", dans le but de fournir des influences et des alliances positives (Hébert, 1987).

Ce projet se situe à la frontière de la prévention primaire et secondaire. La prévention primaire selon la définition du Comité de la santé mentale du Québec (1985b) vise les groupes pour qui aucune hypothèse de risque n'a été formulée (les pairs prosociaux). La prévention secondaire comporte des stratégies qui permettent d'identifier et de traiter le plus tôt possible un désordre de façon à en réduire la durée et la sévérité (Cowen, 1983). L'intervention auprès des pairs agressifs dans ce projet correspond à cette définition.

Le projet comprenait trois activités. Une activité psychocorporelle visait à développer le contrôle de soi par le karaté traditionnel. Une deuxième activité comportait I'animation PRODAS (Programme de développement affectif et social : prévention et résolution de conflits; Palomares et al., 1987). Cette dernière intégrait des discussions de groupe autour de valeurs et de conduites prosociales à adopter dans les situations de la vie courante. De plus, un réseau de soutien social a été organisé auprès de quelques parents des pairs agressifs en vue d'appuyer leurs acquisitions positives et d'assurer le transfert de celles-ci dans la communauté (Hébert, 1987). Trois groupes d'une même école ont été rejoints par le projet.

Nous discuterons dans cet article de l'expérience d'animation (PRODAS) ainsi que des changements observés chez les membres 
(agressifs et prosociaux) du groupe de la $6^{\mathrm{e}}$ année $\mathrm{e}^{1}$. II est à noter qu'il n'a pas été possible de respecter le ratio de trois "prosociaux " pour un " agressif " avec les jeunes de la $6^{\mathrm{e}}$ année. Il y a eu moins de jeunes prosociaux que prévu qui se sont intéressés au projet.

Nous essaierons d'identifier les changements observés en rapport avec les objectifs prévus. Les objectifs du groupe tels que déterminés par le projet pilote (Hébert, 1987) visaient à augmenter le contrôle de soi chez des jeunes agressifs et prosociaux. Le projet tentait aussi de développer et de maintenir des comportements prosociaux dans la communauté ainsi que de diminuer les comportements agressifs jugés destructeurs, en plus d'augmenter l'estime de soi.

Le programme de groupe a été élaboré à partir de PRODAS et s'est échelonné sur 35 rencontres. Toutefois, alors que les 26 premières ont suivi le modèle d'éducation structurée tel que prévu, les 9 dernières ont été consacrées à la planification et à la réalisation d'une action dans le milieu selon le modèle à buts sociaux (Tremblay et Home, 1990).

\section{L'agression chez les jeunes}

L'agression serait définie comme une action où s'exerce un pouvoir transmis par un certain apprentissage cognitif et social. De plus, l'intention de cette action serait jugée socialement destructrice dans un milieu donné (Hébert, 1989). Elle peut se manifester de façon physique ou verbale, directe ou indirecte, elle peut être active ou passive (Buss, 1961). En milieu scolaire elle peut prendre des formes aussi diverses que l'abus verbal, les règlements de compte, les menaces, le harcèlement, l'intolérance et le racisme. La plupart des auteurs concluent que généralement, la manifestation de comportements agressifs en bas âge a des répercussions à l'adolescence et à l'âge adulte. Les comportements agressifs ont tendance à se maintenir dans le temps, de la petite enfance à l'adolescence jusqu'à l'âge adulte. L'enfant agressif est davantage destiné à devenir un adolescent déviant, de même que l'adolescent agressif, un criminel adulte (Loeber et Dishion, 1983; Tremblay, 1987).

L'omniprésence de la violence dans la société témoigne d'une difficulté à vivre des rapports sociaux de manière souple et positive. Les nombreux cas de violence envers les femmes et les enfants témoignent des risques de répercussions de ces comportements agressifs à l'adolescence et à l'âge adulte. Trente mille femmes ont été victimes de violence conjugale en 1985 (La Gazette des femmes, 1986), près de 20000 enfants sont référés chaque année à la DPJ. Plus du quart des vols qualifiés sur le territoire de la CUM et le tiers des abus 
sexuels signalés au Québec ont pour auteurs des jeunes de moins de 18 ans (Chapdelaine, 1989; Presse Canadienne, 1988).

On cite divers facteurs sociaux qui peuvent être associés à l'augmentation de la violence : la densité de population reliée au phénomène urbain, la paupérisation de certaines couches sociales (les jeunes en particulier) et les messages médiatiques particulièrement véhiculés par les films télévisés (Dot, 1984). De plus, le soutien familial s'est modifié. Non seulement on assiste à une diminution de l'influence et du soutien de la famille élargie, nous constatons par ailleurs que la famille nucléaire est de plus en plus éclatée. Depuis 1968, un million de Québécois ont été touchés par le divorce et une famille sur cinq est monoparentale. La majorité d'entre elles vivent de l'aide sociale et font ainsi face aux problèmes de pauvreté tout en cumulant une surcharge de travail. En plus des carences affectives et matérielles dans leur milieu familial, les jeunes sont devenus insécures face aux institutions (religion, école) et ne peuvent plus se raccrocher aux anciennes croyances qui guidaient autrefois les lignes de conduite. La formation académique dispensée est souvent éloignée des préoccupations quotidiennes des étudiants et étudiantes. Peut-être que cohabitent un sentiment d'aliénation dans un système impersonnel et froid, un relâchement de la discipline et l'apathie vécue par le personnel enseignant (DSC Hôpital général de Montréal, 1989; Côté et al., 1988; Comité de la santé mentale au Québec, 1985).

La manifestation de comportements agressifs associés à la violence en milieu scolaire n'est pas un phénomène nouveau; ce qui est nouveau, c'est la volonté des divers intervenants à trouver des moyens et des outils afin de prévenir cette violence, de l'enrayer, ou du moins de l'atténuer. Il est important d'identifier le plus tôt possible les jeunes qui pourraient être perturbés afin de pouvoir établir des programmes préventifs appropriés. Ceci est nécessaire si I'on ne veut pas voir ces comportements problèmes s'établir à l'âge adulte et les individus devenir alors réfractaires aux interventions thérapeutiques (Schwartzman, Serbin et Ledingham, 1987; Edleson, 1981; Robins et Ratcliff, 1979).

\section{Le groupe comme moyen de prévention des comportements agressifs}

\section{Le groupe : un outil de prévention}

Depuis ses débuts, le service social des groupes a été utilisé comme outil de prévention auprès de jeunes vulnérables ou margina- 
lisés. Les travailleuses et travailleurs sociaux de groupe se sont inspirés du modèle à buts sociaux afin de favoriser le développement "normal » des jeunes en plus de mobiliser et de canaliser leur leadership de façon positive. On mettait l'accent sur la participation active des membres non seulement dans le déroulement des activités mais aussi dans les prises de décision en groupe (Klein, 1972). Plus récemment, le modèle d'éducation structurée s'est ajouté au répertoire des intervenants et intervenantes de groupe. Ce modèle vise l'acquisition d'habiletés, par exemple la résolution de conflits, et peut permettre aux jeunes de mieux vivre la transition entre l'enfance et I'adolescence (Drum et Knott, 1977; Bessell, 1987; Palomares et al., 1987). Ce modèle se distingue du courant central en service social des groupes par son accent sur le rôle central de l'animateur ou animatrice qui planifie les activités à l'avance, et par l'importance moindre accordée au processus et à l'évolution du groupe (Papell et Rothman, 1983). L'intervention de groupe qui fait l'objet de cet article s'est basée d'abord sur le modèle d'éducation structurée, auquel s'est ajouté graduellement le modèle à buts sociaux, en réponse à l'évolution du groupe (Tremblay et Home, 1990).

\section{Expériences de prévention des comportements agressifs chez les jeunes}

L'abondance de publications sur le phénomène de la délinquance chez les jeunes prédomine sur celles qui portent sur les comportements agressifs et sur les moyens de prévention. Cet article ne présente que les études et expériences les plus récentes et les plus pertinentes en relation avec notre objet d'étude. Toutes ces expériences utilisent les méthodes de groupe, mais avec des modèles variés. La plupart sont de l'extérieur du service social, utilisant surtout le modèle d'éducation structurée. Les expériences relatées portent sur des interventions auprès de jeunes du préscolaire, de l'élémentaire et du secondaire. Un nombre restreint d'entre elles s'adressent de façon plus spécifique aux garçons (Swerdlik et al. 1978; Tremblay, 1985) et d'autres aux filles (Frey, 1965).

Ces expériences comprennent un programme d'activités qui porte une attention particulière au développement de la coopération. Elles visent aussi à faire prendre conscience aux jeunes des manifestations de la violence, à en comprendre les conséquences et à rechercher des solutions pacifiques à des situations de conflits. Elles mettent l'accent sur la connaissance, l'appréciation de soi et la valorisation sociale (Baril, 1989; Lamontagne et Ouellet, 1988; Rose, 1987). Le programme porte habituellement sur des discussions de groupe, jeux de rôles, activités récréatives et artistiques, sensibilisa- 
tion et activités faisant appel à la participation (Côté et al., 1988), et qui se réalisent autant durant les heures de classe qu'à l'heure du dîner ou après la classe.

Les expériences relatées font mention de plusieurs avantages du travail de groupe comme moyen de prévenir les comportements agressifs. L'apprentissage d'habiletés rend les jeunes capables d'interagir au sein du groupe et fournit une préparation à une participation efficace dans la société en général (Wodarski et Hedrick, 1987; Rose, 1987). Ces expériences permettent de réduire les problèmes que l'on rencontre dans des situations particulières de conflits telles que la frustration et la manipulation. De plus, les activités produisent des conséquences positives comme le respect mutuel et la recherche d'alternatives (Edleson, 1981). Selon Rose (1987), un tel type d'intervention de groupe a des répercussions immédiates et futures sur l'ajustement social des jeunes. De plus, l'acquisition, le développement et la maîtrise de compétences en résolution de conflits contribuent à promouvoir une santé mentale positive (Anderson et Messick, 1974; Shure, 1981). Le groupe peut ainsi devenir un lieu privilégié pour apprendre de nouveaux comportements et développer des ressources nouvelles pour faire face aux conflits (Palomares et al., 1987).

\section{Méthodes d'analyse}

\section{Problèmes d'évaluation de l'intervention de groupe}

Même si les intervenants et intervenantes commencent à reconnaître l'importance d'évaluer leurs programmes de prévention, peu d'entre eux le font de façon systématique (Boivin et Lavoie, 1984). Seulement $10 \%$ des articles publiés en service social des groupes entre 1975 et 1983 présentent les résultats de recherche ou d'évaluation (Feldman, 1986). Diverses raisons peuvent expliquer cette situation, comme le nombre de variables à contrôler (Beaudry, 1984) et la difficulté d'utiliser certaines techniques (groupe contrôle, mesures pré et post test) lorsqu'on évalue une intervention sur le terrain (Brower et Garvin, 1989).

Certains auteurs et auteures ont toutefois identifié des critères à respecter dans l'évaluation des interventions préventives. Boivin et Lavoie (1984) insistent sur la pertinence (et non le caractère expérimental) du devis, et soutiennent que l'évaluation doit avoir recours à des instruments variés. En intervention de groupe, Fike (1980) affirme qu'il faut évaluer non seulement les résultats (individuels ou re. groupés) mais aussi les caractéristiques du groupe ou la satisfaction des membres. 
L'évaluation de notre intervention préventive en groupe a respecté ces principes. Le devis non expérimental prévoyait l'utilisation de plusieurs instruments à différents moments et auprès de diverses personnes pour apprécier le changement chez les membres. De plus, les caractéristiques du groupe et son évolution ont été observées et analysées (Tremblay et Home, 1990).

\section{Devis d'évaluation}

Le tableau 1 présente le devis d'évaluation utilisé afin d'analyser les changements observés chez les membres en rapport avec les objectifs de départ. Nous avons utilisé 4 instruments pour notre évaluation : un questionnaire d'auto-évaluation destiné aux participants et participantes et un questionnaire d'évaluation rempli par leurs parents et leurs professeurs. De plus, l'intervenant a complété un cahier de bord et une grille en rapport avec un profil de développement affectif et social. Le projet global comportait aussi des mesures d'évaluation du comportement et de l'estime de soi, mais nous n'en ferons pas rapport ici'.

\section{Instruments de mesure}

Un questionnaire d'auto-évaluation (Hébert, 1987), comprenant diverses questions sur le déroulement du projet a été administré aux membres à la $15^{\circ}$ et à la $30^{\circ}$ rencontres. Conscients des limites de l'auto-évaluation (subjectivité), nous avons utilisé deux autres sources de données en fournissant un questionnaire d'évaluation aux parents et professeurs des participants et participantes. La collecte de données a été également effectuée à la $15^{\mathrm{e}}$ et $30^{\mathrm{e}}$ rencontres. Des notes d'observation compilées par l'intervenant après chaque réunion, à partir d'un cahier de bord, ont permis de cumuler des données sur le cheminement des membres.

Un profil de développement affectif et social a été élaboré à partir de la grille PRODAS (Bessell, 1987) basée sur l'approche de Champney (1941). Il s'agit d'un ensemble de 6 échelles, soit 2 pour chacun des 3 facteurs : conscience, réalisation et interaction sociale. Elles fournissent une appréciation qualitative et une appréciation quantitative qui permettent de donner des indications sur le comportement et le progrès des participants et participantes tout au long de l'expérience de groupe. Les 6 échelles, conscience de soi, sensitivité* aux autres, confiance en soi, efficience*, compréhension interpersonnelle et, tolérance, ont été évaluées 5 fois, soit à toutes les 6 rencontres $\left(6^{\circ}, 12^{\circ}, 18^{\circ}, 24^{\circ}\right.$ et $30^{\prime \prime}$ rencontres). Chaque échelle comporte 


\section{TABLEAU 1}

Devis d'évaluation des changements observés chez les membres du groupe

\begin{tabular}{|c|c|c|c|c|}
\hline $\begin{array}{c}\text { Objectifs } \\
\text { d'évaluation }\end{array}$ & $\begin{array}{l}\text { Instruments } \\
\text { utilisés }\end{array}$ & Contenu & $\begin{array}{l}\text { Moment de } \\
\text { collecte }\end{array}$ & Population \\
\hline \multirow{4}{*}{ 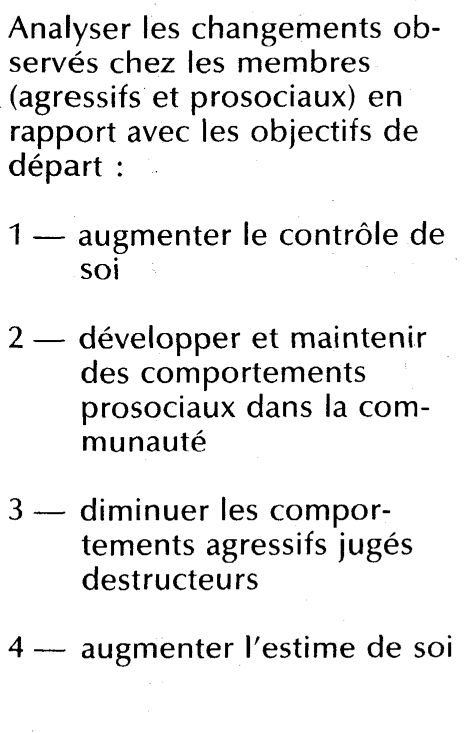 } & $\begin{array}{l}\text { Questionnaire } \\
\text { d'évaluation }\end{array}$ & $\begin{array}{l}\text { Changements } \\
\text { observés et com- } \\
\text { mentaires } \\
\text { généraux }\end{array}$ & $\begin{array}{l}15^{\mathrm{e}} \text { et } 30^{\mathrm{e}} \text { ren- } \\
\text { contres }\end{array}$ & $\begin{array}{l}\text { Professeurs et } \\
\text { parents de tous } \\
\text { les partici- } \\
\text { pant(e)s }\end{array}$ \\
\hline & $\begin{array}{l}\text { Questionnaire } \\
\text { d'auto-évalua- } \\
\text { tion }\end{array}$ & $\begin{array}{l}\text { Changements } \\
\text { observés, satis- } \\
\text { factions, com- } \\
\text { mentaires }\end{array}$ & $\begin{array}{l}15^{e} \text { et } 30^{e} \text { ren- } \\
\text { contres }\end{array}$ & $\begin{array}{l}\text { Tous les par- } \\
\text { ticipant(e)s }\end{array}$ \\
\hline & $\begin{array}{l}\text { Cahier de bord } \\
\text { (observations } \\
\text { participantes) }\end{array}$ & $\begin{array}{l}\text { Observations sur } \\
\text { le cheminement } \\
\text { des participants }\end{array}$ & $\begin{array}{l}\text { Après chaque } \\
\text { rencontre }\end{array}$ & $\begin{array}{l}\text { Tous les par- } \\
\text { ticipant }(e) s\end{array}$ \\
\hline & $\begin{array}{l}\text { Profil de } \\
\text { développement } \\
\text { affectif et social } \\
\text { complété par } \\
\text { l'intervenant }\end{array}$ & $\begin{array}{l}\text { Perception de } \\
\text { l'intervenant du } \\
\text { profil de DAS } \\
\text { (développement } \\
\text { affectif et social) }\end{array}$ & $\begin{array}{l}6^{\mathrm{e}}, 12^{\mathrm{e}}, 18^{\mathrm{e}}, 24^{\mathrm{e}} \text { et } \\
30^{\mathrm{e}} \text { rencontres }\end{array}$ & $\begin{array}{l}\text { Tous les par- } \\
\text { ticipant }(\mathrm{e}) \mathrm{s}\end{array}$ \\
\hline
\end{tabular}


des descriptions précises identifiées par un nombre de 0 à 10. À l'aide de ces descriptions, l'intervenant détermine le nombre qui identifie le mieux la perception qu'il a du participant ou de la participante. II reporte ce nombre sur l'échelle appropriée et, après chaque évaluation, on peut voir une certaine tendance apparaître.

L'échelle conscience de soi fait appel à ce que la personne ressent, pense et fait de façon consciente, alors que la sensitivité aux autres se définit par une attention au bien-être des autres. La confiance en soi s'exprime dans l'acceptation de ce que l'on est, l'envie d'essayer de nouvelles choses tout en étant plus sûr de soi, tandis que l'efficience consiste à faire face aux situations de façon appropriée. La compréhension interpersonnelle détermine comment on évalue, de façon positive ou négative, le comportement d'une personne en rapport avec ce même comportement chez une autre personne. Quant à la tolérance, c'est la capacité de reconnaître et d'accepter les différences individuelles (Bessell, 1987).

\section{Analyse des données}

Notre analyse des données comporte deux étapes. La première a consisté à établir une moyenne générale pour le groupe, à partir du profil de développement affectif et social des membres. Nous avons distingué chacun des sous-groupes, agressifs et prosociaux, en établissant leur moyenne spécifique. Nous sommes conscients qu'il y a une perte de données avec cette méthode, mais elle permet d'apprécier le progrès général du groupe sans perdre les différences entre les sous-groupes. Nous avons aussi effectué une analyse de contenu à partir des questionnaires. Nous avons ressorti les commentaires de différents observateurs qui portaient sur les changements observés lors de l'expérience de groupe. Ensuite nous avons mis en parallèle les observations et les objectifs de départ afin de déterminer lesquels de ces changements permettaient de constater l'atteinte des objectifs. Nous avons effectué cette comparaison en tenant compte des changements observés, d'une part chez les jeunes agressifs et, d'autre part, chez les jeunes prosociaux. Cette analyse de données a été complétée à l'aide du cahier de bord qui comprenait des notes compilées sur le cheminement des membres après chaque rencontre.

\section{Résultats et analyse des changements observés}

Dans cette section nous discuterons des résultats obtenus à l'aide des instruments utilisés et nous analyserons les données qui ont été recueillies en comparant les deux sous-groupes (agressifs et proso- 
ciaux) sous l'angle des changements observés. Nous discuterons d'abord les changements reliés au contrôle et à l'estime de soi (tableau 2), et ensuite ceux reliés aux comportements prosociaux et agressifs (tableau 3 ).

Le tableau 2 montre les changements au niveau du contrôle de soi et de l'estime de soi, observés chez les membres des deux sousgroupes par les professeurs, les parents, les participants et participantes, et l'intervenant. Selon les données compilées dans ce tableau, la plupart des jeunes perçus comme agressifs ont augmenté leur contrôle de soi. Les parents considèrent que leur jeune accepte un peu plus l'autorité et qu'il a acquis un peu plus de retenue dans ses comportements. Les participants et participantes se disent plus patients et plus attentifs envers les autres. Selon l'intervenant, la plupart des jeunes évaluent davantage le pour et le contre avant de poser un geste. Cependant les professeurs nuancent les résultats en affirmant que certains jeunes sont plus calmes alors que d'autres n'ont pas changé, ils continuent à manquer de contrôle. On peut donc supposer que l'intervention a permis d'augmenter le contrôle de soi chez plusieurs d'entre eux. En ce qui concerne les jeunes identifiés comme prosociaux, ceux-ci ont aussi augmenté leur contrôle de soi. Les professeurs et les parents rapportent qu'ils sont plus attentifs, plus calmes et qu'ils ont une meilleure concentration. Cependant l'intervenant considère que les changements ont été moins prononcés chez ces derniers, compte tenu des habiletés qu'ils possédaient déjà. Par contre, on n'a pas assisté à un revirement, du positif au négatif, dans les attitudes ou comportements chez les "prosociaux ". Il s'agit d'un constat intéressant, car ils avaient un " concept de soi " déjà fort et solide.

On a aussi observé des modifications dans l'estime de soi. Une majorité de jeunes agressifs sont devenus plus positifs et plus confiants en eux, aux dires des professeurs et des parents. C'est ce que les jeunes expriment en affirmant qu'ils sont "fiers d'eux " et qu'ils sont " mieux dans leur peau ». L'intervenant constate que les jeunes n'hésitaient pas à parler de leurs réussites, cela de façon plus marquée vers la fin de l'expérience de groupe; en ce sens on peut parler d'une manifestation de l'estime de soi plus grande. Par exemple, les jeunes aimaient parler de la planificaton et de la réalisation de l'activité de clôture comme étant leur idée, rappeler que celle-ci était née de leur initiative. Cette augmentation dans l'estime de soi peut être attribuée au choix du programme et à l'ordre dans lequel les activités ont été présentées. Ces deux facteurs ont favorisé l'acquisition maximale des habiletés que l'on voulait transmettre et ont ainsi facilité l'atteinte des objectifs, comme le préconise l'approche d'éducation structurée (Papell et Rothman, 1983; Drum et 


\section{TABLEAU 2}

Comparaison des objectifs par rapport aux changements observés chez les pairs agressifs et prosociaux (Contrôle de soi et estime de soi)

\begin{tabular}{|c|c|c|c|c|c|}
\hline \multirow{2}{*}{$\begin{array}{c}\text { Objectifs } \\
\text { poursuivis }\end{array}$} & \multirow{2}{*}{$\begin{array}{l}\text { Groupe } \\
\text { cible }\end{array}$} & \multicolumn{4}{|c|}{ Changements observés chez le participant(e)s } \\
\hline & & Professeurs & Parents & Participants & Intervenant \\
\hline \multirow[t]{2}{*}{$\begin{array}{l}\text { Augmenter le } \\
\text { contrôle de } \\
\text { soi }\end{array}$} & $\begin{array}{c}\text { Pairs } \\
\text { agressifs }\end{array}$ & $\begin{array}{l}\text { - certains sont } \\
\text { plus calmes; } \\
\text { d'autres man- } \\
\text { quent de con- } \\
\text { trôle }\end{array}$ & $\begin{array}{l}\text { - acceptent un } \\
\text { peu plus } \\
\text { l'autorité } \\
\text { - un peu plus } \\
\text { de retenue }\end{array}$ & $\begin{array}{l}\text { - plus patients } \\
\text { - plus attentifs }\end{array}$ & $\begin{array}{l}\text { - la plupart } \\
\text { évaluent } \\
\text { davantage le } \\
\text { pour et le } \\
\text { contre avant } \\
\text { de poser un } \\
\text { geste }\end{array}$ \\
\hline & $\begin{array}{c}\text { Pairs } \\
\text { prosociaux }\end{array}$ & $\begin{array}{l}\text { - plus attentifs } \\
\text { - plus calmes } \\
\text { - meilleure } \\
\text { concentra- } \\
\text { tion }\end{array}$ & - plus calmes & $\begin{array}{l}\text { - plus calmes } \\
\text { - plus sérieux }\end{array}$ & $\begin{array}{l}\text { - idem, mais } \\
\text { changements } \\
\text { moins pro- } \\
\text { noncés }\end{array}$ \\
\hline
\end{tabular}




\begin{tabular}{|c|c|c|c|c|c|}
\hline \multirow[t]{2}{*}{$\begin{array}{l}\text { Augmenter } \\
\text { l'estime de } \\
\text { soi }\end{array}$} & $\begin{array}{l}\text { Pairs } \\
\text { agressifs }\end{array}$ & $\begin{array}{l}\text { - quelques-uns } \\
\text { sont plus } \\
\text { positifs }\end{array}$ & $\begin{array}{l}\text { - plus confiants } \\
\text { et plus fiers } \\
\text { d'eux }\end{array}$ & $\begin{array}{l}\text { - « je suis fier } \\
\text { de mon com- } \\
\text { portement } \\
\text { - "je suis } \\
\text { mieux dans } \\
\text { ma peau» }\end{array}$ & $\begin{array}{l}\text { - parlent de } \\
\text { leurs réussites } \\
\text { avec enthou- } \\
\text { siasme }\end{array}$ \\
\hline & $\begin{array}{c}\text { Pairs } \\
\text { prosociaux }\end{array}$ & $\begin{array}{l}\text { - réussissent } \\
\text { mieux, per- } \\
\text { forment et } \\
\text { s'affirment } \\
\text { davantage }\end{array}$ & $\begin{array}{l}\text { - plus de con- } \\
\text { fiance }\end{array}$ & $\begin{array}{l}\text { - aucune re- } \\
\text { marque }\end{array}$ & - idem \\
\hline
\end{tabular}


Knott, 1977). À titre d'exemple, certaines activités visant l'acquisition d'habiletés en résolution de conflits comportaient des jeux de rôles dans lesquels les jeunes expérimentaient de nouveaux comportements face aux conflits. Heap (1987) constate que le fait de se manifester dans un conflit, peu importe que les résultats soient positifs ou négatifs, peut augmenter l'estime de soi. Le fait de participer à ce type d'activité a pu influencer l'estime de soi. Quant aux jeunes prosociaux, les professeurs et les parents disent que ceuxci réussissent mieux, performent et s'affirment davantage tout en ayant acquis plus de confiance. L'intervenant observe les mêmes changements chez ce groupe que chez les "agressifs" alors que les participants et participantes ne font aucune remarque à ce sujet.

Le tableau 3 présente les changements observés dans le développement et le maintien de comportements prosociaux dans la communauté ainsi que dans la diminution des comportements agressifs jugés destructeurs chez les deux sous-groupes. Le développement et le maintien de comportements prosociaux dans la communauté se sont caractérisés, pour les jeunes agressifs, par un plus grand respect des autres, une prise de conscience de leurs actes et une plus grande motivation à bien faire ce qu'ils entreprennent. Les jeunes utilisaient des moyens positifs pour résoudre des conflits, comme le compromis et la négociation (voir Tremblay et Home, 1990). L'exemple qui suit démontre bien le transfert de comportements prosociaux dans la communauté qui a pu se produire suite à l'expérience de groupe. Un jeune perçu comme agressif est devenu coanimateur pour les enfants plus jeunes dans des activités organisées après l'école. Son leadership, d'abord négatif, a été mobilisé et réorienté de sorte que son potentiel soit exploité de façon positive. Cet exemple est étroitement relié à l'utilisation du modèle à buts sociaux, dans la deuxième partie de l'intervention, qui favorise la mobilisation du potentiel et du leadership de chacun (Klein, 1972; Lewis, 1983). Chez les jeunes prosociaux, l'augmentation des comportements prosociaux s'est manifestée par l'aide apportée aux autres et l'utilisation de la discussion pour régler les " chicanes ». Le fait qu'ils acceptaient davantage les idées différentes des leurs, notamment celles des membres dits "agressifs", est identifié par l'intervenant comme la plus importante acquisition de ces jeunes.

Tout en développant des comportements prosociaux, les jeunes ont diminué les comportements agressifs jugés destructeurs. $C^{\prime} e s t$ le cas chez le sous-groupe des "agressifs" dont certains disent : "avant je donnais des coups inutilement", ou encore "je parle à l'autre plutôt que de me battre ». Ils sont moins agressifs en paroles et en gestes selon certains parents. D'ailleurs, l'intervenant a remarqué une diminution des menaces physiques et verbales entre les membres 
(insultes, coups) pendant les réunions. L'opinion est partagée chez les enseignants; selon eux certains jeunes sont plus agressifs, $d$ 'autres moins, et un ne l'est plus. Selon nous, il n'y a aucun doute que ces jeunes ont progressé, mais il est possible que pour certains, ce progrès ait été moins ressenti à l'école.

Cela s'explique en partie par le fait que certains ont bénéficié d'un soutien de leur famille et de l'école, qui s'ajoutait à l'intervention de groupe. Ceci suggère qu'il y aurait eu lieu d'assurer une collaboration plus étroite entre les enseignants et les intervenants du projet afin de maximiser le transfert des acquisitions dans le milieu scolaire pour tous les participants et participantes. C'est ce que préconise l'approche d'éducation structurée comme nous l'avons mentionné précédemment (Papell et Rothman, 1983).

II est cependant plus difficile d'identifier chez les "prosociaux" des changements nous permettant de conclure que les comportements agressifs jugés destructeurs ont diminué. Ces étudiants et étudiantes étaient identifiés au départ comme possédant davantage de comportements positifs et, d'autre part, il y a eu très peu de données fournies par les professeurs et les parents sur ce point. Ce que nous pouvons dire, $c^{\prime}$ est que les professeurs ont observé que quelques-uns étaient plus positifs. Par contre, un parent dit qu'il trouve son enfant agressif sans préciser s'il s'agit d'un changement suite au projet, ou s'il était moins agressif avant. Il est à noter que ce jeune avait été identifié au préalable par le milieu scolaire et familial comme prosocial. Le jeune avait-il été mal identifié par le milieu familial et scolaire, ou a-t-il bel et bien développé des comportements agressifs pendant et après l'expérience? Nous pouvons émettre l'hypothèse que son agressivité découle de facteurs extérieurs à l'expérience de groupe, tels des changements survenus au niveau de la famille et des amis. L'intervenant a aussi observé chez les "prosociaux" la diminution de certaines attitudes de rejet qu'ils avaient auparavant lorsqu'ils dénigraient les idées du sous-groupe "agressif".

Le tableau 4 présente les données obtenues sur les changements observés chez les membres du groupe dans leur développement affectif et social à partir de la grille PRODAS (Bessell, 1987). Ce tableau représente la moyenne des deux sous-groupes selon trois facteurs : conscience, réalisation et interaction sociale, lesquels sont divisés en deux sous-échelles. Dans l'ensemble, on voit que le groupe est parti $d^{\prime}$ un niveau très bas dans tous les facteurs à la $6^{\circ}$ rencontre (2.2) pour atteindre un niveau assez élevé à la $30^{\circ}$ rencontre (6.5). On remarque aussi que des changements importants ne se sont fait sentir qu'à la $18^{\prime \prime}$ rencontre, étape où le groupe commençait à prendre certaines initiatives. Nous n'analyserons pas cet aspect relié à l'évolution du 
TABLEAU 3

Comparaison des objectifs par rapport aux changements observés chez les pairs agressifs et prosociaux (Comportements prosociaux et agressifs)

\begin{tabular}{|c|c|c|c|c|c|}
\hline \multirow{2}{*}{$\begin{array}{l}\text { Objectifs } \\
\text { poursuivis }\end{array}$} & \multirow{2}{*}{$\begin{array}{l}\text { Groupe } \\
\text { cible }\end{array}$} & \multicolumn{4}{|c|}{ Changements observés chez le participant(e)s } \\
\hline & & Professeurs & Parents & Participants & Intervenant \\
\hline \multirow[t]{2}{*}{$\begin{array}{l}\text { Développer et } \\
\text { maintenir des } \\
\text { compor- } \\
\text { tements } \\
\text { prosociaux } \\
\text { dans la com- } \\
\text { munauté }\end{array}$} & $\begin{array}{c}\text { Pairs } \\
\text { agressifs }\end{array}$ & $\begin{array}{l}\text { - se respectent } \\
\text { entre eux } \\
\text { - prennent } \\
\text { conscience de } \\
\text { leurs actes }\end{array}$ & $\begin{array}{l}\text { - plus motivés } \\
\text { à bien faire ce } \\
\text { qu'ils entre- } \\
\text { prennent }\end{array}$ & $\begin{array}{l}\text { - plus respec- } \\
\text { tueux (s'ex- } \\
\text { cuser, se don- } \\
\text { ner la main, } \\
\text { pardonner, } \\
\text { etc.) }\end{array}$ & $\begin{array}{l}\text { - utilisent } \\
\text { moyens posi- } \\
\text { tifs pour } \\
\text { résoudre con- } \\
\text { flits (com- } \\
\text { promis, etc.) }\end{array}$ \\
\hline & $\begin{array}{c}\text { Pairs } \\
\text { prosociaux }\end{array}$ & $\begin{array}{l}\text { - aucune re- } \\
\text { marque }\end{array}$ & $\begin{array}{l}\text { - ont amélioré } \\
\text { leur compor- } \\
\text { tement }\end{array}$ & $\begin{array}{l}\text { - apportent } \\
\text { aide aux } \\
\text { autres } \\
\text { - discutent } \\
\text { pour régler } \\
\text { chicane }\end{array}$ & $\begin{array}{l}\text { - acceptent } \\
\text { plus les idées } \\
\text { différentes } \\
\text { des leurs }\end{array}$ \\
\hline
\end{tabular}




\begin{tabular}{|c|c|c|c|c|c|}
\hline \multirow[t]{2}{*}{$\begin{array}{l}\text { Diminuer les } \\
\text { comporte- } \\
\text { ments agres- } \\
\text { sifs jugés des- } \\
\text { tructeurs }\end{array}$} & $\begin{array}{c}\text { Pairs } \\
\text { agressifs }\end{array}$ & $\begin{array}{l}\text { - certains sont } \\
\text { plus agressifs, } \\
\text { d'autres } \\
\text { moins, et un } \\
\text { a cessé d'être } \\
\text { agressif }\end{array}$ & $\begin{array}{l}\text { moins agres- } \\
\text { sifs en } \\
\text { paroles et en } \\
\text { gestes }\end{array}$ & $\begin{array}{l}\text { - « avant je } \\
\text { donnais des } \\
\text { coups inu- } \\
\text { tilement", } \\
\text { "parle à } \\
\text { l'autre plutôt } \\
\text { que de me } \\
\text { battre " }\end{array}$ & $\begin{array}{l}\text { menaces ver- } \\
\text { bales et phy- } \\
\text { siques ont } \\
\text { diminué (in- } \\
\text { sultes, coups, } \\
\text { etc.) }\end{array}$ \\
\hline & $\begin{array}{c}\text { Pairs } \\
\text { prosociaux }\end{array}$ & $\begin{array}{l}\text { - quelques-uns } \\
\text { sont plus } \\
\text { positifs }\end{array}$ & $\begin{array}{l}\text { - un parent } \\
\text { trouve son } \\
\text { enfant agres- } \\
\text { sif }\end{array}$ & $\begin{array}{c}\text { — se parler } \\
\text { pour régler } \\
\text { un conflit } ~\end{array}$ & $\begin{array}{l}\text { - menaces ver- } \\
\text { bales ont } \\
\text { diminué }\end{array}$ \\
\hline
\end{tabular}




\section{TABLEAU 4}

\section{Profil de développement affectif et social des membres du groupe \\ (Moyenne du groupe)}

\begin{tabular}{|l|l|l|l|l|l|}
\hline \multirow{2}{*}{ Facteurs } & \multicolumn{5}{|c|}{ Rencontres } \\
\cline { 2 - 6 } & $\mathbf{6}^{\mathbf{e}}$ & $\mathbf{1 2}^{\mathbf{e}}$ & $\mathbf{1 8}^{\mathbf{e}}$ & $\mathbf{2 4}^{\mathbf{e}}$ & $\mathbf{3 0}^{\mathbf{e}}$ \\
\hline $\begin{array}{l}\text { Conscience } \\
\text { Conscience de soi } \\
\text { Sensitivité }\end{array}$ & 2.6 & 3.1 & 4.4 & 5.9 & 6.4 \\
\hline $\begin{array}{c}\text { Réalisation } \\
\text { Confiance en soi } \\
\text { Efficience }\end{array}$ & 2.4 & 2.6 & 4.3 & 5.9 & 6.7 \\
\hline $\begin{array}{c}\text { Interaction sociale } \\
\text { Compréhension } \\
\text { interpersonnelle }\end{array}$ & 2.1 & 2.6 & 4.9 & 5.5 & 6.4 \\
Tolérance & 1.9 & 3.1 & 4.6 & 6.7 & 6.7 \\
\hline $\begin{array}{c}\text { Moyenne selon les } \\
\text { rencontres }\end{array}$ & $\mathbf{2 . 2}$ & $\mathbf{2 . 8}$ & $\mathbf{4 . 2}$ & $\mathbf{5 . 7}$ & $\mathbf{6 . 5}$ \\
\hline
\end{tabular}

groupe qui a été discuté dans un article précédent (Tremblay et Home, 1990). Une autre constatation est que les six échelles semblent avoir évolué de façon constante et selon le même rythme. Les résultats qui suivent vont nous permettre d'apprécier les changements observés selon les deux sous-groupes. De plus, ils favoriseront la comparaison des différences entre les groupes d'après les six échelles de la grille PRODAS.

Le tableau 5 présente le facteur de conscience avec ses deux sous-échelles, conscience de soi et sensitivité. On peut percevoir une nette différence dès le départ entre les deux sous-groupes. On note à l'échelle conscience de soi le faible niveau (1.6) avec lequel le sousgroupe des "agressifs" a débuté en comparaison avec celui des " prosociaux » (3.2) par rapport au niveau qu'il a atteint à la $30^{\mathrm{e}}$ rencontre (6.7). D'ailleurs, à cette échelle, les changements ont été plus 


\section{TABLEAU 5}

\section{Comparaison des moyennes des pairs agressifs/prosociaux}

\begin{tabular}{|l|l|l|l|l|l|c|}
\hline \multirow{2}{*}{ Facteur de conscience } & \multicolumn{5}{|c|}{$\begin{array}{c}\text { Change- } \\
\text { ments } \\
\text { selon les } \\
\text { sous- } \\
\text { groupes }\end{array}$} \\
\cline { 2 - 7 } & $\mathbf{6}^{\mathbf{e}}$ & $\mathbf{1 2}^{\mathbf{e}}$ & $\mathbf{1 8}^{\mathbf{e}}$ & $\mathbf{2 4}^{\mathbf{e}}$ & $\mathbf{3 0}^{\mathbf{e}}$ & \\
\hline $\begin{array}{l}\text { Conscience de soi } \\
\text { Pairs agressifs }\end{array}$ & 1.6 & 3.2 & 4.2 & 6 & 6.7 & +5.1 \\
$\quad$ Pairs prosociaux & 3.2 & 3 & 4.7 & 5.8 & 6.5 & +3.3 \\
\hline $\begin{array}{l}\text { Sensitivité } \\
\text { Pairs agressifs }\end{array}$ & 1 & 2.7 & 4 & 5.5 & 6.3 & +5.3 \\
Pairs prosociaux & 2.6 & 2.3 & 4.7 & 6.2 & 7 & +4.4 \\
\hline
\end{tabular}

prononcés $(+5.1)$ chez les " agressifs " que chez les "prosociaux" $(+3.3)$. Le même phénomène s'est produit à l'échelle de sensitivité où les " agressifs" ont débuté au niveau 1 et terminé à celui de 6.3 par rapport aux " prosociaux » qui sont passés du niveau 2.6 au niveau 7 . De plus ces changements se sont fait sentir plus tôt chez les " agressifs " (12 rencontre) alors qu'à ce moment les " prosociaux " enregistraient une légère régression pour atteindre un véritable changement à la $18^{\mathrm{e}}$ rencontre. Ici encore, il est important de mentionner que c'est à cette étape que les membres ont vraiment commencé à agir " ensemble " comme groupe, au sens où l'entend Schwartz (1971).

Le tableau 6 démontre que les résultats par rapport à la confiance en soi et à l'efficience donnent un portrait similaire aux résultats précédents. Les deux sous-groupes débutent avec une faible cote, mais plus élevée pour les "prosociaux". Cependant, les changements se produisent en même temps pour les deux sous-groupes, à la différence que les "agressifs " acquièrent davantage de confiance en soi et sont plus efficients à la $24^{\mathrm{e}}$ rencontre, alors que chez les "prosociaux» on pouvait déjà remarquer cette tendance à la $18^{\mathrm{e}}$ rencontre. Nous croyons que ces deux aspects s'acquièrent alors que les jeunes sont stimulés par des tâches dont le niveau de difficulté est peu élevé, ce qui assure le succès (Bessell, 1987). Nous croyons 


\section{TABLEAU 6}

\section{Comparaison des moyennes des pairs agressifs/prosociaux}

\begin{tabular}{|c|c|c|c|c|c|c|}
\hline \multirow{2}{*}{ Facteur de réalisation } & \multicolumn{5}{|c|}{ Rencontres } & $\begin{array}{c}\text { Change- } \\
\text { ments } \\
\text { selon les } \\
\text { sous- } \\
\text { groupes }\end{array}$ \\
\cline { 2 - 8 } & $\mathbf{6}^{\mathbf{e}}$ & $\mathbf{1 2}^{\mathbf{e}}$ & $\mathbf{1 8}^{\mathbf{e}}$ & $\mathbf{2 4}^{\mathbf{e}}$ & $\mathbf{3 0}^{\mathbf{e}}$ & \\
\hline $\begin{array}{l}\text { Confiance en soi } \\
\text { Pairs agressifs }\end{array}$ & 1.7 & 2.6 & 3.2 & 5 & 6 & +4.3 \\
Pairs prosociaux & 2.8 & 3 & 4.7 & 6 & 6.7 & +3.9 \\
\hline $\begin{array}{l}\text { Efficience } \\
\text { Pairs agressifs }\end{array}$ & & & & & & \\
Pairs prosociaux & 1.3 & 2.6 & 3.5 & 5.5 & 6.7 & +5.4 \\
& 2.6 & 2.7 & 4.7 & 5.8 & 6.8 & +4.2 \\
\hline
\end{tabular}

que c'est ce qui s'est produit pour les deux sous-groupes mais à des moments différents. Selon Henry (1981), le programme en service social doit être planifié afin de favoriser la réussite des buts recherchés par les membres aux différents moments dans l'expérience de groupe.

Le tableau 7 présente le facteur d'interaction sociale qui comporte deux sous-échelles : compréhension interpersonnelle et tolérance. Au niveau de la tolérance, les changements se font sentir pour les deux sous-groupes à la $18^{\circ}$ rencontre; par contre, la cote des "prosociaux" a très peu augmenté $(0.4)$ entre la $6^{\circ}$ et la $12^{\text {e }}$ rencontres, comparativement aux « agressifs» (1.2). Une autre différence se situe au niveau de la compréhension interpersonnelle alors que les changements ont commencé à être plus importants à la $12^{\mathrm{c}}$ rencontre pour les "prosociaux» et à la 18 " pour les "agressifs".

\section{Conclusion}

L'intervention de groupe discutée dans cet article avait pour objectif premier d'augmenter le contrôle et l'estime de soi chez des jeunes agressifs et prosociaux. Elle visait aussi à développer et à 


\section{TABLEAU 7}

\section{Comparaison des moyennes des pairs agressifs/prosociaux}

\begin{tabular}{|c|c|c|c|c|c|c|}
\hline \multirow{2}{*}{$\begin{array}{l}\text { Facteur d'interaction } \\
\text { sociale }\end{array}$} & \multicolumn{5}{|c|}{ Rencontres } & \multirow{2}{*}{$\begin{array}{c}\text { Change- } \\
\text { ments } \\
\text { selon les } \\
\text { sous- } \\
\text { groupes }\end{array}$} \\
\hline & $6^{e}$ & $12^{\mathrm{e}}$ & $18^{\mathrm{e}}$ & $24^{\mathrm{e}}$ & $\mathbf{3 0}^{\mathrm{e}}$ & \\
\hline \multicolumn{7}{|l|}{$\begin{array}{l}\text { Compréhension } \\
\text { interpersonnelle }\end{array}$} \\
\hline Pairs agressifs & 1.3 & 2.7 & 4.2 & 6 & 6.7 & +5.4 \\
\hline Pairs prosociaux & 2.8 & 3.7 & 5 & 6.4 & 6.8 & +4.0 \\
\hline \multicolumn{7}{|l|}{ Tolérance } \\
\hline Pairs agressifs & 1 & 2.2 & 3.7 & 5 & 6 & +5.0 \\
\hline Pairs prosociaux & 2.4 & 2.8 & 4 & 5.6 & 6.3 & +3.9 \\
\hline
\end{tabular}

maintenir des comportements prosociaux dans la communauté ainsi qu'à diminuer les comportements agressifs jugés destructeurs. Nous avons observé des changements positifs en rapport avec chacun de ces objectifs. De plus, les changements ont été plus prononcés chez les jeunes perçus comme agressifs que chez les jeunes identifiés comme prosociaux. Globalement, nos observations permettent de constater que ces jeunes, en particulier les "agressifs", sont plus attentifs au bien-être des autres et qu'ils font face aux situations de conflits de façon plus appropriée. Même s'il ne faut pas généraliser les résultats à cause du petit nombre de sujets observés, les données suggèrent que ce type $d^{\prime}$ intervěntion de groupe auprès des jeunes peut être prometteur. II faudrait que d'autres chercheurs répètent cette expérience avec des échantillons plus grands, afin de vérifier l'efficacité de cette forme d'intervention.

Le type de population visée, des jeunes perçus comme agressifs jumelés avec des jeunes identifés comme prosociaux, nous laisse supposer qu'il faut prévoir une intervention échelonnée sur plusieurs mois, voire une année. II est important que ce soit répétitif dans le temps pour que les jeunes en retirent le maximum de bénéfices. Selon les expériences, les effets bénéfiques ne se font sentir qu'après 
20 semaines de rencontres (Palomares et al., 1987). D'ailleurs, le groupe n'a vraiment commencé à changer qu'à la $18^{\text {e }}$ rencontre.

II est important aussi que ce type de groupe soit ancré dans le quotidien pour faciliter le transfert d'apprentissage (Home, 1980). Ainsi les membres peuvent profiter de la vie de tous les jours pour essayer des changements, et revenir au groupe régulièrement pour recevoir rétroaction et soutien. Ceci n'est possible que lorsqu'on prévoit des réunions au moins une fois par semaine pendant plusieurs mois.

Même si les traditions du service social des groupes avec les jeunes préconisent des interventions d'assez longue durée, les pratiques actuelles vont dans le sens des interventions à court terme. Cette tendance semble être plus appropriée aux groupes de soutien et de transition qui s'adressent d'habitude à des populations homogènes et dont l'autonomie est plus grande. Par contre notre groupe s'adressait à une population hétérogène et peu autonome, ce qui demande un plus grand investissement dans le temps pour atteindre les objectifs fixés (Lang, 1972). Chez certaines clientèles difficiles à rejoindre, il faut prévoir beaucoup de temps avant d'observer des modifications importantes de comportements ou d'attitudes (Breton, 1979). De plus, des recherches suggèrent que certains changements, par exemple ceux liés à l'estime de soi, peuvent avancer assez vite dans un groupe alors que d'autres, tels les changements de comportements, peuvent prendre plus de temps, même dans les groupes composés de personnes sans problèmes particuliers (Home, 1980).

II faut que les travailleuses et travailleurs sociaux résistent aux pressions d'intervenir en groupe uniquement pour des raisons économiques (Henry, 1981). Ils doivent aussi refuser de faire des groupes à court terme lorsque ceux-ci risquent de répondre davantage aux besoins de l'organisme qu'à ceux des membres. Les décisions quant à la durée, au type de programme et d'approche à utiliser doivent se fonder plutôt sur une étude de la clientèle et du contexte (Home et Darveau-Fournier, 1980). Il est à noter que ce projet a pu fonctionner entre autres grâce à la présence de stagiaires et du chercheur principal, ce qui a réduit l'investissement financier requis de l'organisme. Les clients tels que des jeunes agressifs n'ont pas développé leurs difficultés du jour au lendemain, il ne faut donc pas s'attendre à ce qu'ils résolvent leurs problèmes instantanément. $\mathrm{Si}$ on leur accorde le temps nécessaire, ils vont progresser significativement, à leur rythme. 


\section{Notes}

' Pour plus de détails sur les résultats et l'analyse du projet global, il faudrait se reporter à la thèse de Hébert (1990) : La prévention de l'agression juvénile : un projet-pilote. (à paraître).

* Nous avons repris les catégories énoncées par Bessell (1987) et choisi, pour les besoins de cet article, les termes " sensitivité " et " efficience " qui nous semblent rendre avec plus de justesse l'idée que l'auteur voulait transmettre.

\section{Références}

ANDERSON, S. et S. MESSICK (1974). "Social competency in young children ", Developmental Psychology, vol. 10 : 282-293.

BARIL, D. (1989). "Quatre exemples d'interventions visant à prévenir la violence à l'école ", Apprentissage et socialisation, vol. 12, $\mathrm{n}^{\circ} 1: 5-8$.

BeAUDRY, J. (1984). "L'évaluation de programme " : 389-415, dans B. GAUTHIER (éd.), Recherche sociale : de la problématique à la collecte de données. Québec : P.U.Q.

BesseLL, H. (1987). Le développement socio-affectif de l'enfant. Montréal : Actualisation, IDH inc.

BoIviN, M. et F. LAvOIE (1984). "Éléments à considérer dans l'évaluation de programme de prévention en milieu scolaire ", Psychologie Canadienne, vol. $25, \mathrm{n}^{\circ} 2: 105-114$.

BRETON, M. (1979). "Nurturing abused and abusive mothers : The hair dressing group ", Social Work With Groups, vol. 2, n 2 : 161-173.

BROWER, A. et C. GARVIN (1989). "Design issues in social group work research ", Social Work With Groups, vol. 12, n' 3 : 91-102.

Buss, A.H. (1961). The psychology of aggression. New York : Willey.

ChAMPEY, H. (1941). "The measurement of parent behavior ", Child Development, vol. $42, \mathrm{n}^{\circ} 2: 131-168$.

Chapdelaine, B. (1989). "Plan d'action pour contrer la criminalité chez les jeunes", La Presse, vol. 105, n 309, cahier A15.

COMITÉ DE LA SANTÉ MENTALE DU QUÉBEC (1985). La santé mentale des enfants et des adolescents: vers une approche plus globale. Gouvernement du Québec : Division des publications gouvernementales.

COMITÉ DE LA SANTÉ MENTALE DU QUÉBEC (1985 b). La santé mentale : prévenir, traiter et réadaptër efficacement : l'efficacité de la prévention. Gouvernement du Québec : Division des publications gouvernementales.

Côté, G., M. Leblanc, G. Ouellet et A. Pronovost (1988). Violence et délinquance à l'école: inventaire des mesures préventives. Montréal : Services des études, CECM.

COWEN, E.L. (1983). "Primary prevention in mental health : past, present and future ", dans R.D. Felner, L.A. Jason, J.N. Moritsugu, S.S. Farber (éd.), Preventive psychology : theory, research and practice. New York: Pergamon Press.

Dot, O. (1984). Agressivité et violence chez l'enfant et l'adolescent. Verviers : Les Nouvelles Éditions Marabout. 
DRUM, D.J. et J.E. KNOTT (1977). Structured groups for facilating development. New York: Human Sciences Press.

D.S.C. hÔPITAL GÉNÉRAL DE mONTRÉAL (1989). Prévention de la violence en milieu scolaire : Guide d'animation. Montréal : Département de Santé Communautaire, Hôpital Général de Montréal.

EDLESON, J.L. (1981). "Teaching children to resolve conflict: a group approach ", Social Work, vol. 26, n $6: 488-493$.

FELDMAN, R. (1986). "Group work knowledge : A two-decade comparaison", Social Work With Groups, vol. 9, $\mathrm{n}^{\circ} 3$ : 7-14.

FIKE, D.F. (1980). "Evaluating group intervention ", Social Work With Groups, vol. 3, $\mathrm{n}^{\circ} 2: 41-51$

FREY, L.A. (1965). " A social groupwork approach to socially disadvantaged girls in a school ", Child Welfare, vol. 44, n' $10: 563-569$.

HeAP, K. (1987). La pratique du travail social avec des groupes. Paris : Éditions ESF.

HenRY, S. (1981). Group skills in social work: A four dimensional approach. Itasca, Illinois : Peacock.

HÉBERT, J. (1987). L'utilité d'une activité psychocorporelle d'auto-contrôle et de soutien social auprès de jeunes agressifs et prosociaux : une expérience dans la communauté. Projet de thèse de doctorat, École de service social, Université de Montréal : inédit.

HÉBERT, J. (1989). "La problématique des jeunes agressifs : des points de repère ", Apprentissage et socialisation, vol. 12, $\mathrm{n}^{\circ} 1:$ 45-52.

Home, A. (1980). "Étude exploratoire de quatre types de changements survenus chez les membres de groupes féminins de conscientisation ", Service social, vol. 29, $\mathrm{n}^{\text {os }} 1-2: 152-181$.

Home, A. et L. Darveau-Fournier (1980). "La spécificité du service social des groupes ", Service social, vol. $29, \mathrm{n}^{\text {os }} 1-2: 16-31$.

KLEIN, A. (1972). Effective group work: An introduction to principle and method. New York : Association Press.

Lamontagne, A. et G. Ouellet (1988). La prévention de la violence et de la délinquance à l'école. Activités complémentaires, $3^{\mathrm{e}}$ guide. Montréal : Services des études, CECM.

LANG, N. (1972). "A broad-range model of practice in the social work group ", Social Services Review, vol. 46, n' 1 : 76-82.

LEWIS, E. (1983). "Le service social des groupes dans la vie communautaire : caractéristiques des groupes' et du rôle du travailleur social ", Service social, vol. 32, $\mathrm{n}^{\text {os }} 1-2: 32-49$.

LOEBER, R. et T. DISHION (1983). "Early predictors of male delinquency: a review ", Psychological Bulletin, vol. 94, n 1 : 69-99.

Palomares, U., J. LalanNe et B. LoGan (1987). Programme de développement affectif et social : Prévention et résolution de conflits. Montréal : Actualisation, IDH Inc.

PAPell, C. et B. Róthman (1983). "Le modèle du courant central du service social des groupes en parallèle avec la psychothérapie et l'approche de groupe structurée ", Service social, vol. 32, nos 1-2 : 11-31.

Papell, C. et B. Rothman (1978). "Editorial policy statement ", Social Work With Groups, vol. 1, $\mathrm{n}^{\circ} 1: 1-3$. 
Presse Canadienne (1988). "Les jeunes de moins de 18 ans sont responsables du tiers des abus sexuels signalés au Québec ", La Presse, vol. 105, $\mathrm{n}^{\circ} 1$, cahier A14.

Robins, L.N. et K.S. RATCLIFF (1979). "Risk factors in the continuation of childhood antisocial behavior into adulthood", International Journal of Mental Health, vol. 7 : 96-116.

ROSE, S. (1987). "The development of problem-solving skills in children's groups ", Social Work With Groups, vol. 10, n० 1 : 85-95.

SChwartz, W. et S. Zalba (1971). The practice of group work. New York: Columbia University Press.

Schwartzman, A.E., L.A. Serbin et J.E. Ledingham (1987). "L'agressivité et le retrait social chez les enfants à l'école primaire »: 129-153, dans R.E. Tremblay (éd.), Les comportements agressifs : perspective développementale, intergénérationnelle et interculturelle. Montréal : Université de Montréal, Groupe de recherche interuniversitaire sur la prévention de I'inadaptation psychosociale.

SHURE, M.B. (1981). "Social competence as a problem-solving skill »: 158-185, dans J.D. Wine et M.D. Smye (éd.), Social competence. New York: Guilford Press.

SPAIN, C. (1986). "Un groupe de parents d'adolescents : son cheminement, de l'éducation structurée au modèle à buts sociaux ", Service social, vol. 35, $\mathrm{n}^{\circ} 3: 352-365$.

SWERDLIK, M., W. RICE et E. LARSON (1978). "The effect of group therapy experience in fifth grade acting out boys", School Social Work Journal, vol. $2, \mathrm{n}^{\circ} 2: 83-88$.

TREMBLAY, R.E. (1985). La prévention des comportements anti-sociaux chez les garçons agressifs à l'école primaire: un projet pilote. Vaucresson :

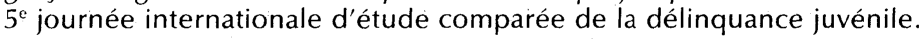

TREMBLAY, R.E. (1987). Les comportements agressifs : perspective développementale, intergénérationnelle et interculturelle. Montréal : Université de Montréal, Groupe de recherche interuniversitaire sur la prévention de l'inadaptation psychosociale.

Tremblay, Y. et A. Home (1990). "De l'éducation à l'action : évolution d'un groupe de jeunes en milieu scolaire ", Intervention, $n^{\circ} 85: 20-32$.

WODARSKI, J. et M. HEDRICK (1987). "Violent children : A practice paradigm ", Social Work in Education, vol. 10, $\mathrm{n}^{\circ} 1: 28-40$. 